\title{
Measurement of Dislocation Density Change during Tensile Deformation in Coarse-Grained Aluminum by In-Situ XRD Technique with Tester Oscillation*1
}

\author{
Hiroki Adachi ${ }^{1}$, Hiroshi Mizowaki ${ }^{1, * 2}$, Masahiro Hirata ${ }^{1, * 3}$, Daisuke Okai ${ }^{1}$ and Hidetaka Nakanishi ${ }^{2}$ \\ ${ }^{1}$ Graduate School of Engineering, University of Hyogo, Himeji 671-2280, Japan \\ ${ }^{2}$ UACJ Corporation Research \& Development Division, Nagoya 455-8670, Japan
}

By conducting in-situ XRD measurement during tensile deformation while oscillating the tensile tester, it was possible to measure the change in dislocation density of a pure aluminum alloy having coarse grains with the grain size of $20 \mu \mathrm{m}$. In the coarse-grained material, the dislocation density during tensile deformation changed through four regions, as in the case of the fine-grained material. Since the dislocation multiplication start stress was very low at $22 \mathrm{MPa}$, the elastic deformation region was very short. Thereafter, the dislocations multiplied rapidly, but when the stress and dislocation density reached $33 \mathrm{MPa}$ and $1.57 \times 10^{14} \mathrm{~m}^{-2}$, respectively, the dislocation multiplication rate was greatly reduced. This is considered to be due to the low dislocation density required to progress the deformation by plastic deformation in coarse-grained aluminum. [doi:10.2320/matertrans.L-M2020861]

(Received August 27, 2020; Accepted October 23, 2020; Published December 4, 2020)

Keywords: dislocation density, grain size, synchrotron radiation, in-situ XRD

\section{Introduction}

Fine-grained alloys with a crystal grain size of less than a few micrometers are reported to exhibit unique mechanical properties that are significantly different from coarse-grained alloys with a grain size of more than $10 \mu \mathrm{m}$ that are conventionally used in industry. For example, the yield strength of pure aluminum with fine grains of less than a few micrometers is much higher than that of coarse-grained aluminum indicated by the Hall-Petch curve. This is called the extra-hardening phenomenon. ${ }^{1)}$ Aluminum alloys typically show continuous yielding; however, in the region where the grain size is less than a few micrometers, the yield point drop phenomenon occurs. ${ }^{1)}$ Contrary to the conventional coarse-grained material, the $0.2 \%$ yield stress of fine-grained aluminum is reduced by cold working and is increased by annealing. This is called the hardening by annealing and softening by deformation phenomenon. ${ }^{2)}$

We have conducted in-situ X-ray diffraction (XRD) measurements using synchrotron radiation (SPring-8) on ultrafine-grained pure nickel with a grain size of $50 \mathrm{~nm}$ and fine-grained pure aluminum with a grain size of $200 \mathrm{~nm}$ to several micrometers, and the change in dislocation density during tensile deformation was investigated. ${ }^{3-7)}$ The change in the dislocation density during tensile deformation was thereby divided into four regions according to the deformation mechanism. Region I is the elastic deformation region where the dislocation density does not increase significantly. In Region II, dislocations begin to multiply rapidly and plastic deformation occurs. However, the dislocation density is not sufficiently large; therefore, elastic deformation also occurs. When the dislocation density $\rho_{\mathrm{II}}$, which allows deformation to proceed only by plastic deformation, is reached, the multiplication of dislocations suddenly slows down and shifts to region III of plastic

\footnotetext{
${ }^{* 1}$ This Paper was Originally Published in Japanese in J. JILM 70 (2020) 274-280.

${ }^{* 2}$ Undergraduate Student, School of Engineering, University of Hyogo

${ }^{* 3}$ Graduate Student, Graduate School of Engineering, University of Hyogo
}

deformation. In Region IV, the dislocation density decreases sharply due to stress unloading associated with fracture. This suggests that the dislocation density measured in the unloaded sample is much lower than the dislocation density during deformation, and that the dislocation structures during deformation and after unloading are different. The results of in-situ measurements thus play an important role in accurately understanding the mechanical properties of finegrained materials.

On the other hand, most of the industrially used structural aluminum alloys are coarse-grained materials with a grain size of more than $10 \mu \mathrm{m}$. It is also important to understand the peculiarities of the mechanical properties of fine-grained aluminum. However, the change in dislocation density in coarse-grained materials by in-situ XRD measurement using synchrotron radiation has not been investigated to date.

The Williamson-Hall method is often used to obtain the dislocation density by XRD measurement, and it is necessary to measure the diffraction angles and half widths of multiple diffraction peaks with this method. ${ }^{8,9)}$ Therefore, to measure the time change of dislocation density in-situ, it is necessary to construct a measurement system that can simultaneously measure over a wide diffraction angle with high angular resolution and high time resolution. The authors have realized this using high-flux synchrotron radiation at SPring-8, as a wide-range detector made by connecting six one-dimensional MYTHEN detectors in series. However, in this experimental system, the one-dimensional detector cuts off a part of the diffraction pattern to obtain a onedimensional diffraction profile. When the number of grains in the X-ray scattering volume of the sample is large, such as in fine-grained materials, the diffraction pattern becomes a continuous Debye ring, so a smooth diffraction profile can be obtained even if part of it is cut out. However, when the number of crystal grains in the X-ray scattering volume is small, such as in coarse-grained materials, the diffraction pattern becomes a discontinuous spot, so a smooth diffraction profile cannot be obtained when a part of it is cut out. Synchrotron radiation has much more directivity and less spread than X-rays emitted from X-ray tubes. As a result, the 
diffraction conditions are stricter and it is difficult for diffraction to occur. Furthermore, it is difficult to increase the beam size of synchrotron radiation; therefore, the scattering volume cannot be increased, and the number of grains that satisfy the diffraction condition is extremely small for coarse-grained materials, and the diffraction tends to be spot-like rather than a Debye-ring.

In a typical X-ray diffraction experiment, the scattering volume is expanded by oscillating the sample, and the number of crystal grains that satisfy the diffraction condition is increased. In this study, a tensile test piece undergoes in-situ XRD measurement during deformation; therefore, we have attempted to solve the problem by oscillating the tensile test piece together with the tensile tester. The purpose of this study was to search for oscillation conditions that could be used to obtain continuous Debye ring-like diffraction and to measure the change in the dislocation density during tensile deformation of coarse-grained aluminum alloy.

\section{Experimental}

Table 1 shows the composition of the A1200 alloy test material used in this work (provided by UACJ Corporation). After casting the A1200 alloy, it was homogenized at $550^{\circ} \mathrm{C}$ for $6 \mathrm{~h}$, hot-rolled from $30 \mathrm{~mm}$ to $2 \mathrm{~mm}$ at $550-200^{\circ} \mathrm{C}$, and cold-rolled to a plate thickness of $0.4 \mathrm{~mm}$. Various heat treatments were applied to this rolled plate to coarsen the grain size, as given in Table 2. The plate was also subjected to accumulative roll bonding (ARB) ${ }^{1)}$ which is a severe

Table 1 Chemical composition of the sample.

\begin{tabular}{|c|c|c|c|c|}
\hline \multicolumn{1}{|c|}{ (mass\%) } \\
\hline $\mathrm{Si}$ & $\mathrm{Fe}$ & $\mathrm{Ti}$ & $\mathrm{Cu}, \mathrm{Mg}, \mathrm{Mn}, \mathrm{Cr}, \mathrm{Zn}$ & $\mathrm{Al}$ \\
\hline 0.05 & 0.86 & 0.02 & $<0.01$ & 99.04 \\
\hline
\end{tabular}

Table 2 Sample name and annealing conditions.

\begin{tabular}{|l|l|}
\hline \multicolumn{1}{|c|}{ Sample name } & \multicolumn{1}{c|}{ Annealing condition } \\
\hline CR & Cold-rolled \\
\hline CRA523 & CR $+448 \mathrm{~K}, 0.5 \mathrm{~h}+523 \mathrm{~K}, 0.5 \mathrm{~h}$ \\
\hline CRA623 & $\mathrm{CR}+448 \mathrm{~K}, 0.5 \mathrm{~h}+623 \mathrm{~K}, 0.5 \mathrm{~h}$ \\
\hline CRA673 & $\mathrm{CR}+673 \mathrm{~K}, 0.5 \mathrm{~h}$ \\
\hline CRA723 & $\mathrm{CR}+723 \mathrm{~K}, 0.5 \mathrm{~h}$ \\
\hline CRA773 & $\mathrm{CR}+773 \mathrm{~K}, 0.5 \mathrm{~h}$ \\
\hline CRA773L & $\mathrm{CR}+773 \mathrm{~K}, 3 \mathrm{~h}$ \\
\hline ARB & $\mathrm{CR}+\mathrm{ARB} 6 \mathrm{cycles}$ \\
\hline ARB373 & ARB $+373 \mathrm{~K}, 0.5 \mathrm{~h}$ \\
\hline ARB423 & ARB $+423 \mathrm{~K}, 0.5 \mathrm{~h}$ \\
\hline ARB448 & ARB $+448 \mathrm{~K}, 0.5 \mathrm{~h}$ \\
\hline ARB498 & ARB373+498K, $0.5 \mathrm{~h}$ \\
\hline ARB523 & ARB373+523K, $0.5 \mathrm{~h}$ \\
\hline ARB573 & ARB373+573K, $0.5 \mathrm{~h}$ \\
\hline ARB623 & ARB373+623K, $0.5 \mathrm{~h}$ \\
\hline
\end{tabular}

plastic deformation process, for 6 cycles to refine the crystal grains. The method involves repeated joining by rolling with a rolling reduction of $50 \%$, by which an infinitely large strain can theoretically be introduced. The ARB samples were subjected to the heat treatment shown in Table 2 to control the grain size. The assigned sample names are given in Table 2. The grain size of these samples was measured by electron back-scattered diffraction (EBSD) using orientation imaging microscopy (OIM) software (TSL) in conjunction with a scanning electron microscope (JSM-6500F, JEOL). Tensile test pieces were cut out using an electric discharge machine so that the rolling direction (RD) was parallel to the tensile direction. The parallel part has a length of $20 \mathrm{~mm}$, a width of $5 \mathrm{~mm}$, and a thickness of $1 \mathrm{~mm}$; Fig. 1 shows the shape of the tensile test piece in detail.

In-situ XRD measurements were performed using the BL19B2 beamline, which uses a bending magnet beam instead of an undulator beam with strong directivity, to increase the number of grains that satisfy the diffraction condition. The energy of the incident X-ray used was $25 \mathrm{keV}$, and the beam size was $0.15 \mathrm{~mm}$ long and $3 \mathrm{~mm}$ wide. A $1 \mathrm{~mm}$ thick A1200 alloy test piece was attached to a small tensile tester mounted on a goniometer. To investigate the appropriate oscillation conditions for in-situ XRD measurements in coarse-grained samples, a two-dimensional detector (PIALTSU-300K, DECTIS) was installed at a distance of $705.32 \mathrm{~mm}$ away from the samples, $13^{\circ}$ above the transmission direction, as shown in Fig. 2; the (111) and (200) diffraction peaks were observed. The distance from the sample to the detector was obtained from the diffraction position of a $\mathrm{CeO}_{2}$ standard sample (National Institute of Standards and Technology; NIST) with a known lattice constant. The time of oscillation in the tensile axis direction (Z-direction) was fixed at $10 \mathrm{~s}$ for 1 cycle, and the change in the Debye ring was measured by changing the oscillation

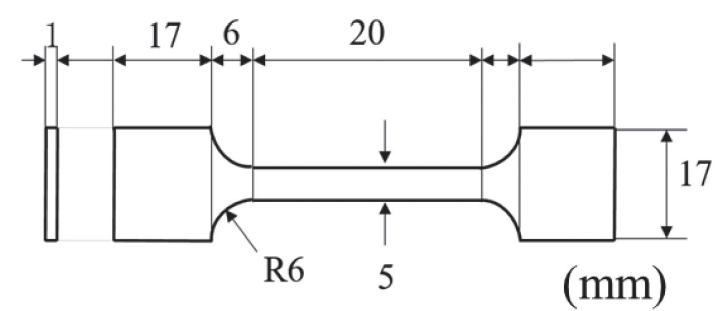

Fig. 1 Dimensions of tensile test pieces.

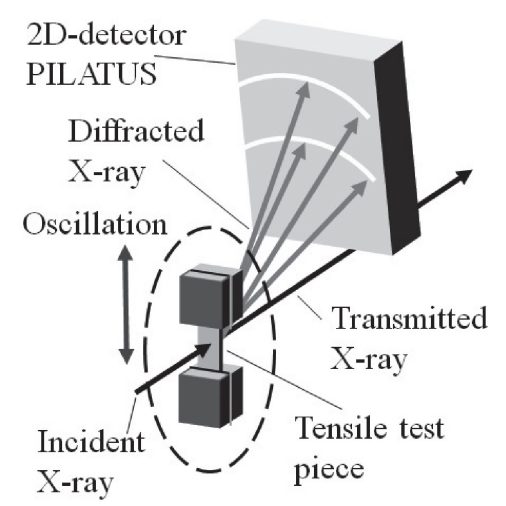

Fig. 2 Schematic diagram of XRD measurements. 


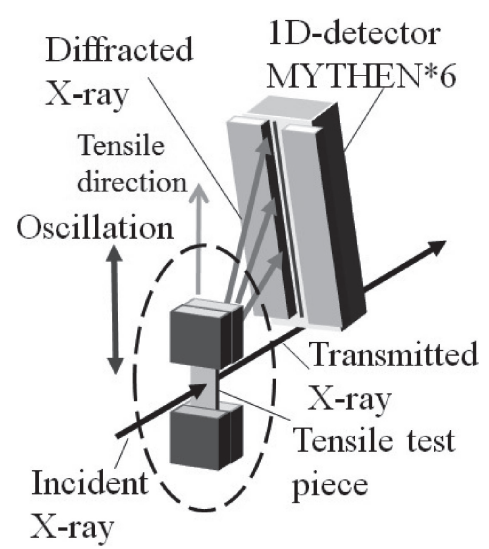

Fig. 3 Schematic diagram of in-situ XRD measurements during tensile test with oscillation.

distance to $0,1,2,3$, and $4 \mathrm{~mm}$. A 6-unit MYTHEN detector was installed $21^{\circ}$ above the transmission direction with a camera length was $704.66 \mathrm{~mm}$ for the in-situ XRD measurement to obtain the dislocation density, as shown in Fig. 3. The camera distance was obtained from the diffraction position of the NIST $\mathrm{CeO}_{2}$ standard sample. An initial strain rate of $3.3 \times 10^{-4} \mathrm{~s}^{-1}$ was applied to the test piece attached to the tensile tester installed on the goniometer. The specimen was subjected to tensile deformation while oscillating in the $\mathrm{Z}$ direction at an oscillation speed of $1 \mathrm{~mm} / \mathrm{s}$ and a width of $2 \mathrm{~mm}$. The measurement was performed with a time resolution of $2 \mathrm{~s}$. The diffraction peak angle and full width at half maximum were calculated from the observed diffraction peak profiles from the (111), (200), (220), (311), (222), and (331) planes, and the dislocation density was calculated using the Williamson-Hall method. ${ }^{4,8,9)}$

\section{Results and Discussion}

Figures 4 and 5 show EBSD measurement results for the ARB and CR773L (see Table 2) materials, respectively. The average grain size, d, was defined as the large-angle grain boundary spacing in the normal direction (ND) on the RDND plane, which was $d=505 \mathrm{~nm}$ for the ARB sample. The grain size increased continuously with the annealing temperature and time, and was coarsened to $\mathrm{d}=3.54 \mu \mathrm{m}$ in the ARB623 material. Table 3 shows the grain sizes obtained by the various annealing processes. From these results, an A1200 alloy with a grain size of $500 \mathrm{~nm}$ to $20 \mu \mathrm{m}$ was prepared.

Figure 6(a) shows XRD results for the CR773L sample, which had the coarsest grain size, obtained with a twodimensional detector without oscillation and an exposure time of $5 \mathrm{~s}$. The direction from the right to the left of the sample was parallel to the tension direction, so that the inside diffraction was from the (111) plane, and the outside diffraction was from the (200) plane. Spot-like diffractions were observed in coarse-grained samples such as those from CR773L. In contrast, ring-shaped diffractions were observed in the ARB sample, as shown in Fig. 6(b), which had fine grains, even when no oscillation was performed. It should be noted that there are two strips in the horizontal direction in the lower part and upper part of Fig. 6 where no diffraction
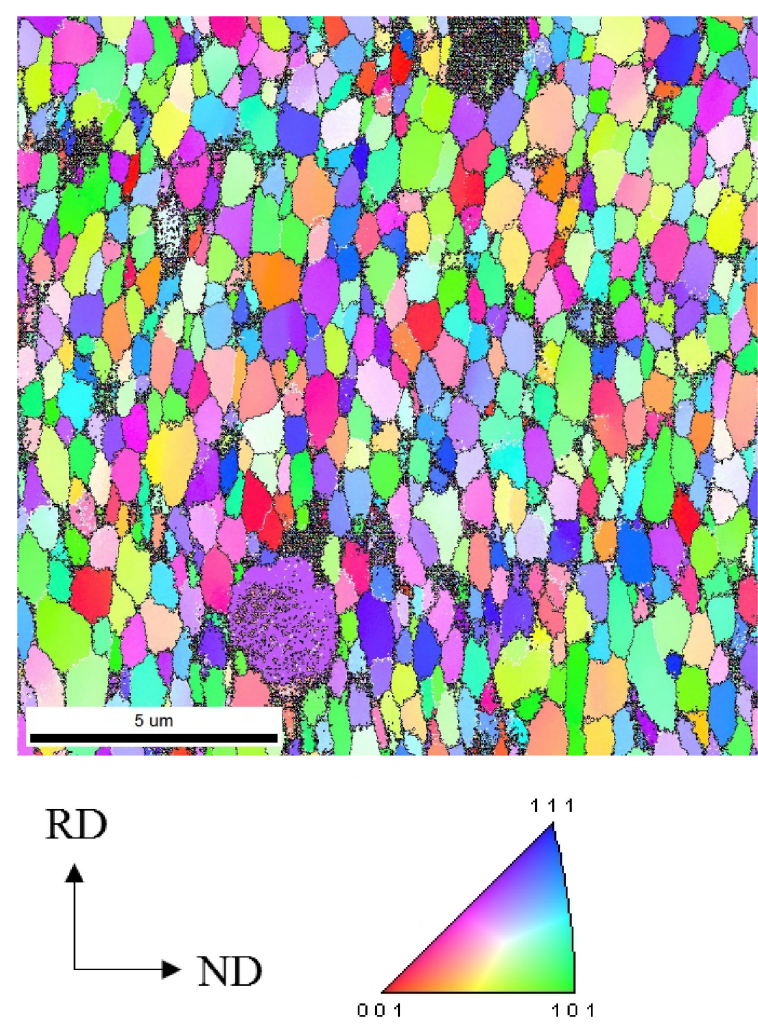

Fig. 4 TD inverse pole figure map of ND-RD plane obtained by EBSD measurement of ARB specimen.
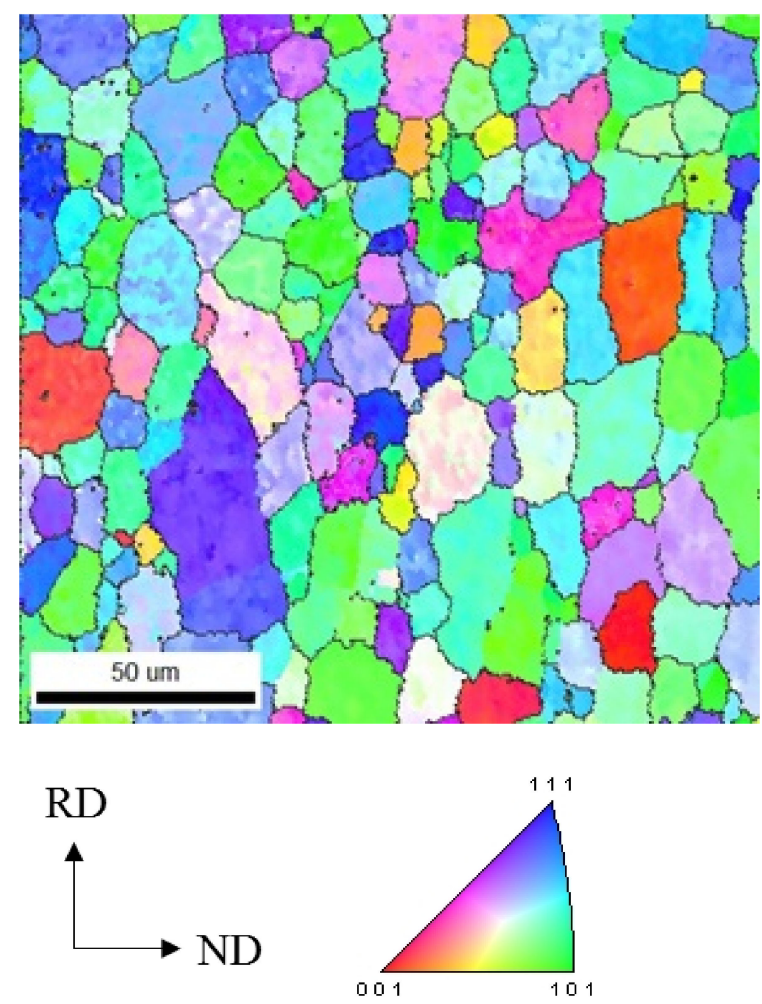

Fig. 5 TD inverse pole figure map of ND-RD plane obtained by EBSD measurement of CR773L specimen.

was detected, which is due to the joint of three detectors, so that there are two strip-shaped connection parts.

Figure 7 shows the azimuth change of the (111) plane diffraction intensity from point $\mathrm{A}$ (azimuth angle $60^{\circ}$ ) to 
Table 3 Average grain diameter of annealed samples.

\begin{tabular}{|c|c|c|}
\hline Annealing condition & Sample name & Average grain diameter $(\mu \mathrm{m})$ \\
\hline As-ARBed & ARB & 0.505 \\
\hline 373K 0.5h & ARB373 & 0.566 \\
\hline 423K 0.5h & ARB423 & 0.635 \\
\hline 448K 0.5h & ARB448 & 0.711 \\
\hline 373K 0.5h + 498K 0.5h & ARB498 & 0.977 \\
\hline 373K 0.5h + 523K 0.5h & ARB523 & 1.26 \\
\hline 373K 0.5h + 573K 0.5h & ARB573 & 3.02 \\
\hline 373K 0.5h + 623K 0.5h & ARB623 & 3.54 \\
\hline 448K 0.5h + 523K 0.5h & CR523 & 1.83 \\
\hline 448K 0.5h $+623 \mathrm{~K} 0.5 \mathrm{~h}$ & CR623 & 10.1 \\
\hline 673K 0.5h & CR673 & 10.1 \\
\hline $723 \mathrm{~K} 0.5 \mathrm{~h}$ & CR723 & 17.5 \\
\hline $773 \mathrm{~K} 0.5 \mathrm{~h}$ & CR773 & 20.2 \\
\hline $773 \mathrm{~K} 3.0 \mathrm{~h}$ & CR773L & \\
\hline
\end{tabular}
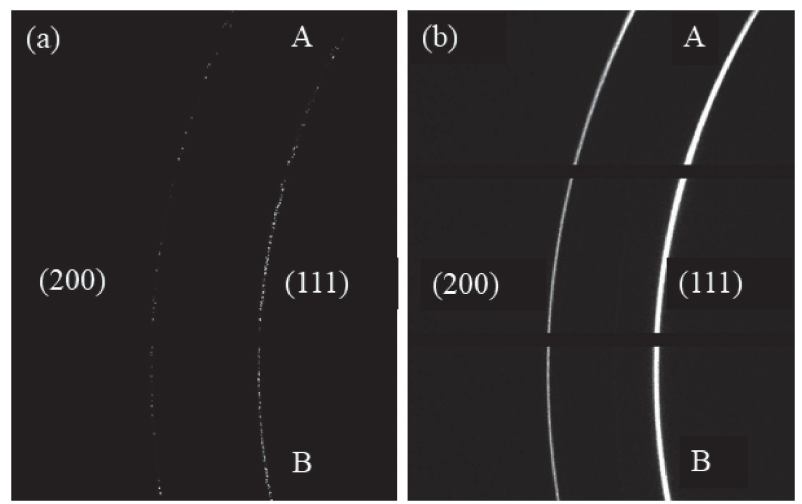

Fig. 6 (111) and (200) diffractions of (a) CR773L and (b) ARB sample measured by two-dimensional detector.

point $\mathrm{B}$ (azimuth angle $100^{\circ}$ ) in Fig. 6. In the ARB material with fine grains, almost constant strength was observed in the vicinity of azimuth angles of $70^{\circ}$ to $100^{\circ}$. Note that the azimuth angle of $90^{\circ}$ is parallel to the tensile direction. The diffraction intensity decreases as the azimuth angle becomes smaller than $70^{\circ}$. This is due to flattening of the beam shape ( $3 \mathrm{~mm}$ wide and $0.15 \mathrm{~mm}$ thick), so that the strength

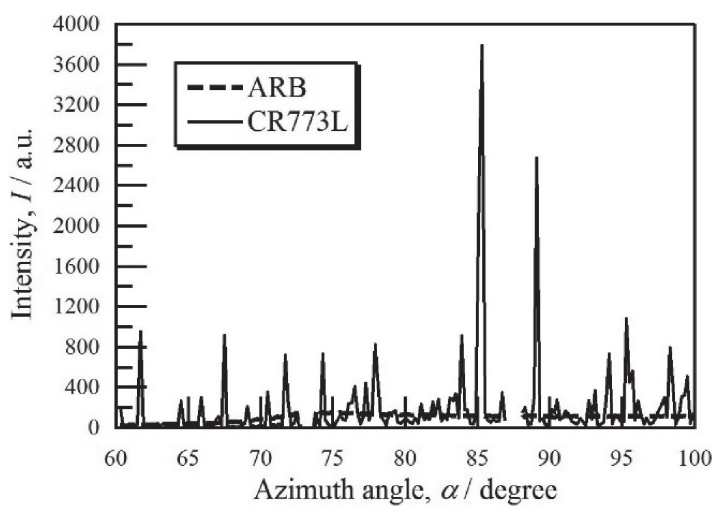

Fig. 7 Change in (111) diffraction intensity along the azimuth direction in $\mathrm{ARB}$ and CR773L samples.

decreases as the azimuth angle moves away from $90^{\circ}$, and also due to the influence of the texture. On the other hand, the diffraction was spot-shaped in the CR773L sample, which indicates there was a large variation in intensity depending on the azimuth angle. From these results, when a onedimensional detector is placed at a position with an azimuth angle of $90^{\circ}$, as shown in Fig. 3, it is expected that a smooth diffraction profile can be obtained with fine-grained material, even without oscillation, whereas the diffraction intensity becomes discontinuous and a smooth profile cannot be obtained with a coarse-grained sample.

Figure 8 shows the change in the diffraction pattern of the CR773L sample depending on the oscillation width. The diffraction increased from the spot shape to the ring shape due to the increase of the oscillation width, even if the exposure time was same. Figure 9 shows the effect of the oscillation width on the change in the (111) plane diffraction intensity in the azimuth direction. The change in the azimuth angle of the diffraction intensity of the CR773L sample was smoothed due to the increase of the swing width, and the strength distribution approached that of the ARB sample with fine grains shown in Fig. 7. Assuming that the crystal grains have a cubic shape with a side length of $20 \mu \mathrm{m}$, where the beam diameter was $0.15 \times 3 \mathrm{~mm}$ and the test piece is $1 \mathrm{~mm}$ thick, there are ca. $5.6 \times 10^{4}$ grains in the scattering volume. When this is oscillated by $1 \mathrm{~mm}$, the number of grains present in the scattering volume increases up to ca. $4.3 \times 10^{5}$, and the number of grains that satisfy the diffraction condition increases eight-fold. When the swing width is increased to 2, 3 , and $4 \mathrm{~mm}$, the number of grains increases to ca. $8.1 \times 10^{5}$,
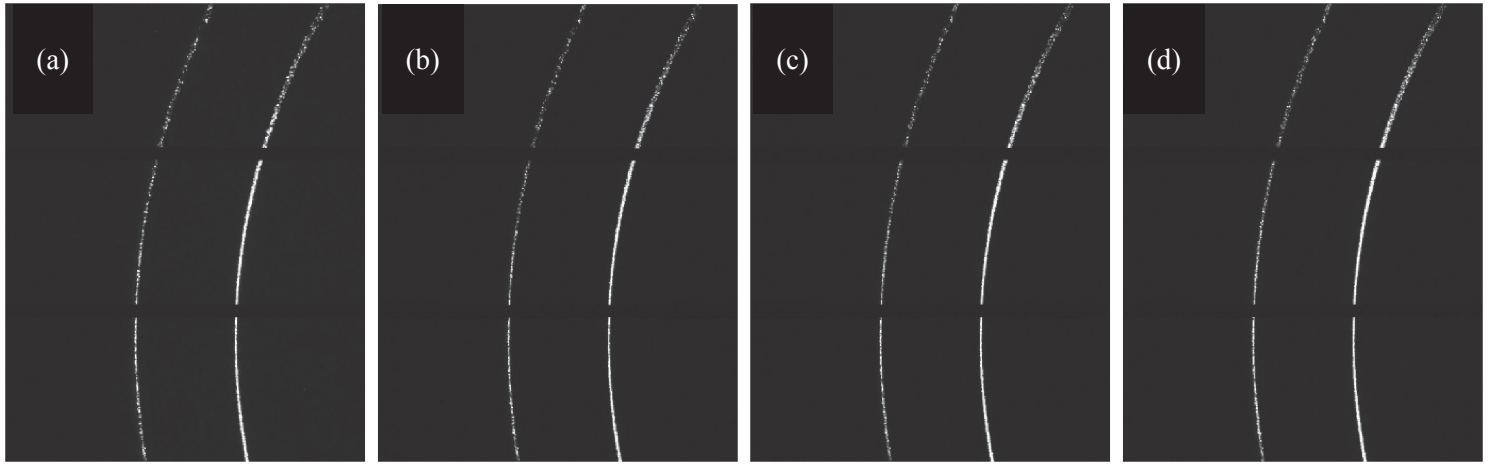

Fig. 8 Change in Debye rings with change in oscillation width in CR773L. (a) $1 \mathrm{~mm}$, (b) $2 \mathrm{~mm}$, (c) $3 \mathrm{~mm}$ (d) $4 \mathrm{~mm}$. 


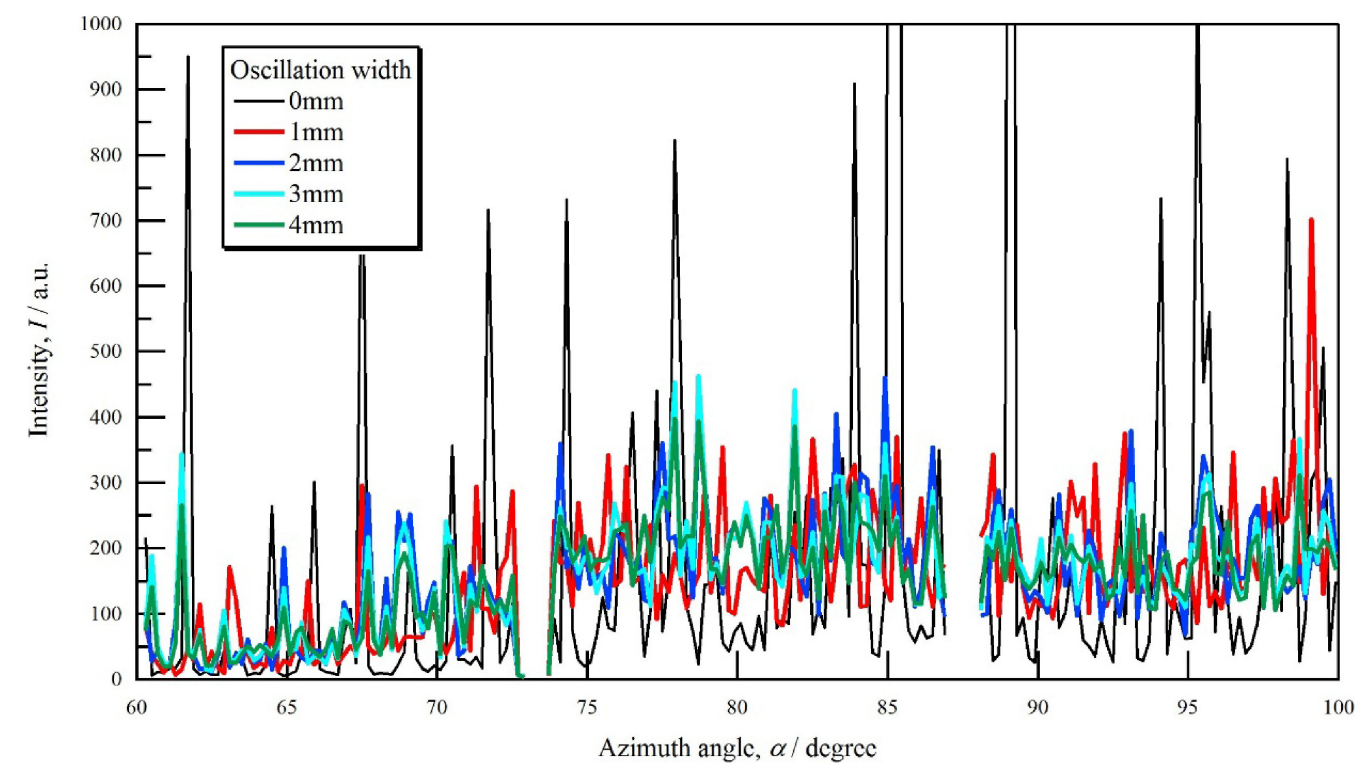

Fig. 9 Change in (111) diffraction intensity along the azimuth direction in ARB and CR773L samples and its changes due to the oscillation width.

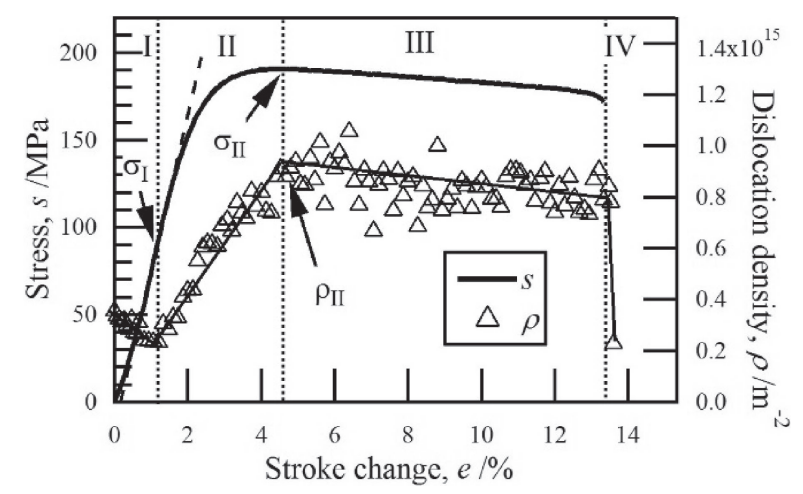

Fig. 10 Change in stress and dislocation density by stroke change in ARB sample.

$1.18 \times 10^{6}$, and $1.56 \times 10^{6}$, respectively and the obtained diffraction profile is expected to be smoothed.

Even if the oscillation was larger than $2 \mathrm{~mm}$, the change in diffraction intensity was small. Therefore, when obtaining the change in the dislocation density during the tensile test using the one-dimensional detector, the in-situ XRD measurement was performed while performing an oscillation with a width of $2 \mathrm{~mm}$. The oscillation speed was then set to $1 \mathrm{~mm} / \mathrm{s}$ to obtain a time resolution of $2 \mathrm{~s}$.

Figure 10 shows the changes in the nominal stress and dislocation density by the stroke change during tensile deformation in the ARB sample. In the fine-grained ARB sample, the dislocation density changed through the four regions (I to IV) with the progress of deformation, as in previous reports. In Region I, the dislocation density did not increase, and the stress increased linearly with the stroke; therefore, this is considered to be an elastic deformation region. The dislocation density increased rapidly when the stress exceeded $\sigma_{\mathrm{I}}$ and shifted to region II; therefore, it is considered that the dislocation source was activated and dislocations multiplied, so that plastic deformation due to dislocation slip began. Although the stress increased slightly more slowly than in the linear relationship between stress and stroke in region I, elastic deformation also occurred because the stress still increased significantly. This is therefore a region where both elastic deformation and plastic deformation occur. The stress $\sigma_{\mathrm{I}}$ at the boundary between region I and region II is $102 \mathrm{MPa}$, which is the stress at which dislocation multiplication starts, and can thus be regarded as the yield stress. The $0.2 \%$ proof stress is $162 \mathrm{MPa}$, and $\sigma_{\mathrm{I}}$ is $60 \mathrm{MPa}$ less than the $0.2 \%$ proof stress; therefore, it is not appropriate to regard the $0.2 \%$ proof stress as the dislocation growth initiation stress in fine-grained samples. The dislocation density increased significantly in region II, and when the dislocation density $\rho_{\text {II }}\left(=9.1 \times 10^{14} \mathrm{~m}^{-2}\right)$ was reached, the change in the dislocation density suddenly became gradual and transitioned to region III. The stress when $\rho_{\text {II }}$ was reached was $\sigma_{\mathrm{II}}=190 \mathrm{MPa}$, so that the $0.2 \%$ proof stress was closer to $\sigma_{\text {II }}$ rather than $\sigma_{\mathrm{I}}$. The details of $\rho_{\mathrm{II}}$ and $\sigma_{\mathrm{II}}$ are discussed later. The change in the dislocation density in region III was very gradual. In pure $\mathrm{Ni}$ with a grain size of $260 \mathrm{~nm}$ prepared by ARB processing, the dislocation density gradually increased in region III, ${ }^{4)}$ so that dislocation multiplication also occurred in this region; however, the melting points of $\mathrm{Ni}$ and $\mathrm{Al}$ are different, so that the dynamic recovery rate is different. It is considered that the dislocation density did not increase in region III, because pure aluminum the dynamic recovery is much faster and dislocation multiplication were balanced. In region IV, the dislocation density decreased sharply due to the unloading that accompanied fracture. This is probably because dislocations disappeared in pairs, or in the case of fine-grained samples, disappeared using grain boundaries as sinks. The significant reduction in the dislocation density by unloading suggests that the dislocation density and dislocation structure observed with an electron microscope after unloading are significantly different from the dislocation density during deformation in the fine-grained sample. This is considered to be one of the major factors that has hindered an accurate understanding of the unique mechanical properties of fine-grained samples. 

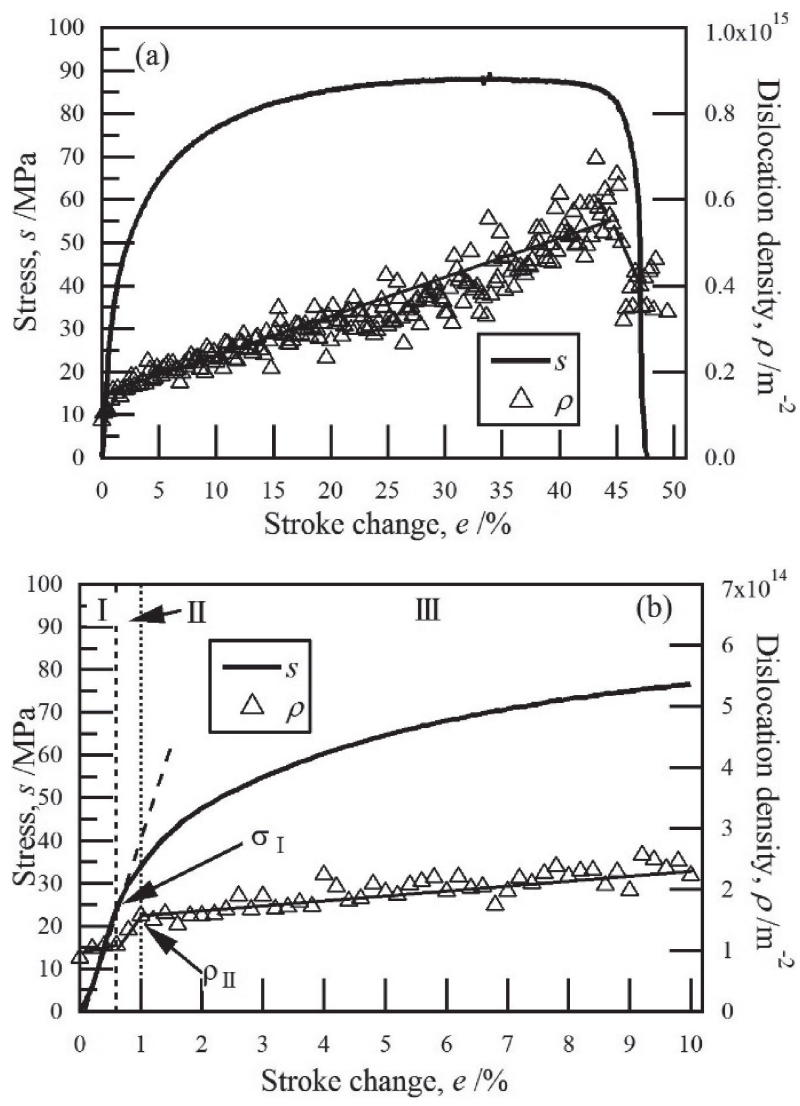

Fig. 11 (a) Change in stress and dislocation density by stroke change in CR773L sample, (b) Enlarged view in the low stroke area of Fig. 11(a).

Figures 11(a) and 11(b) show the changes in nominal stress and dislocation density during tensile deformation in the CR773L sample. It is possible to measure the dislocation density in-situ during deformation by oscillation, even in coarse-grained sample with a grain size of $20 \mu \mathrm{m}$, whereby the dislocation density was determined to change through the four regions as in the case of the fine-grained ARB sample. The stress at which dislocation growth started was very low at $\sigma_{\mathrm{I}}=22 \mathrm{MPa}$, and region I was very short. If the grain size is large, then the maximum length of the Frank-Read source of dislocations that can be activated in the grains becomes long, and the stress for activation of the dislocation source is inversely proportional to the length of the Frank-Read source. ${ }^{10)}$ This is probably because the minimum stress required for dislocation multiplication is low.

Region II, where dislocations proliferate rapidly, was also very short, as was region I. The dislocation density and stress at the transition from region II to III were $\rho_{\text {II }}=1.57 \times$ $10^{14} \mathrm{~m}^{-2}$ and $\sigma_{\mathrm{II}}=33 \mathrm{MPa}$, respectively, and $\rho_{\mathrm{II}}$ was approximately an order of magnitude smaller than that of the ARB sample. There was no significant difference between $\sigma_{\text {II }}$ and $\sigma_{\text {I }}$ and they were much smaller than the ARB sample. Therefore, it is considered that the presence of region II has not been previously considered in detail. The $0.2 \%$ proof stress was $34 \mathrm{MPa}$, which is almost the same as $\sigma_{\mathrm{II}}$, and there was no significant difference from $\sigma_{\mathrm{I}}$, as compared with the fine-grained sample. In the case of coarse-grained materials, even if the $0.2 \%$ proof stress can be regarded as the initiation stress of dislocation growth.

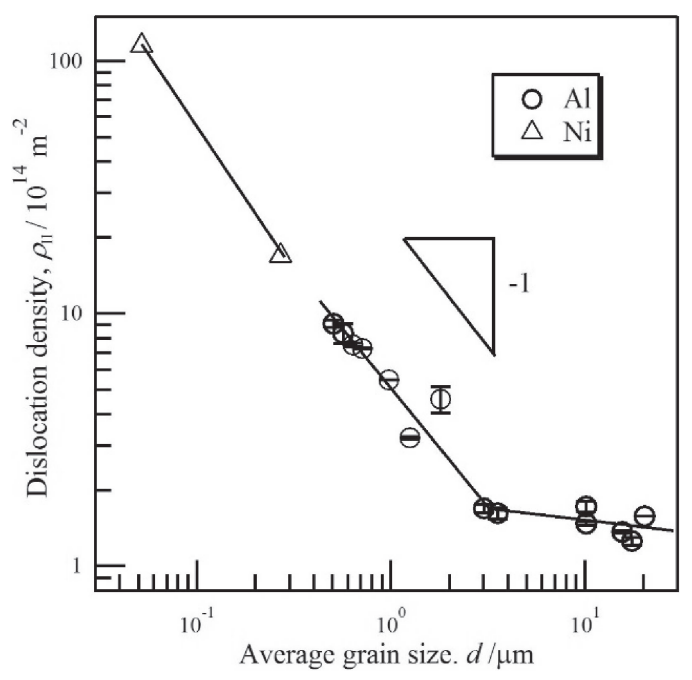

Fig. 12 Change in $\rho_{\text {II }}$ with grain size.

In region III, the dislocation density monotonically increased with the stroke and reached $6 \times 10^{14} \mathrm{~m}^{-2}$, which was approximately four times the initial dislocation density just before fracture. In Region IV, the dislocation density decreased due to the unloading accompanied with fracture; however, the extent of decrease was approximately half of the amount increased in region III. Although the amount of decrease is not small, it is considered that the dislocation structure of the sample after unloading is not so different from that during deformation in the coarse-grained sample, because it is smaller than that in fine-grained materials such as the ARB sample.

Figure 12 shows the change in the crystal grain size of $\rho_{\mathrm{II}}$ in the A1200 alloy and also shows the dependence of $\rho_{\text {II }}$ on the grain size in nanocrystalline $\mathrm{Ni}^{4}{ }^{4)}$ This $\rho_{\mathrm{II}}$ is almost constant at ca. $10^{14} \mathrm{~m}^{-2}$ when $\mathrm{d}>3 \mu \mathrm{m}$, but increases in proportion to the particle size to the power of -1 when $\mathrm{d}<3 \mu \mathrm{m}$. This can be understood by considering the effect of grain size on the mean free path length, $\mathrm{x}$, of dislocations. Shear strain, $\gamma$, generated by plastic deformation is expressed as $^{11)}$

$$
\gamma=\rho \mathrm{bx}
$$

The mean free path length of dislocations is at most about the grain size; therefore, it is considered that the smaller grain size results in a smaller length. ${ }^{12)}$ Assuming that $\mathrm{x}$ is proportional to the grain size $\mathrm{d}$, the dislocation density must increase in proportion to the grain size to the power of -1 to produce the same plastic shear strain. The dislocation density $\rho_{\text {II }}$ required for plastic deformation to occur at a certain constant rate increased in proportion to the grain size to the power of -1 as the grain size became finer. Until the dislocation density $\rho_{\text {II }}$ required for plastic deformation is reached, dislocations must multiply rapidly, and an insufficient amount of deformation is compensated for by elastic deformation, which results in a large increase in stress, although not as much as in region I. This is what happens in Region II; when $\rho_{\text {II }}$ is reached, deformation occurs only by plastic deformation, so that the transition to region III occurs, the dislocation multiplication slows down, and the increase in stress slows down. The stress $\sigma_{\text {II }}$ when showing $\rho_{\text {II }}$ can thus 


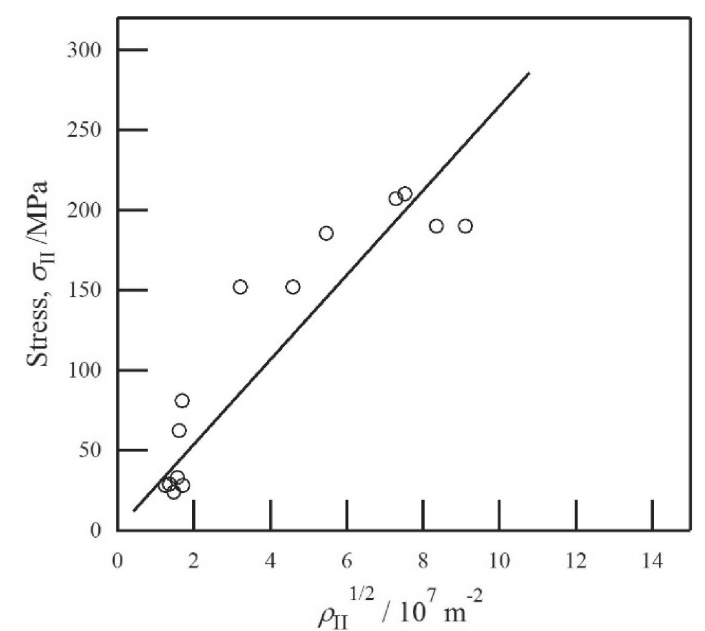

Fig. 13 Relationship between $\sigma_{\mathrm{II}}$ and $\rho_{\mathrm{II}}$ in A1200 alloy with various grain size.

be regarded as a yield stress with a physical meaning different from that of the start of the dislocation multiplication stress $\sigma_{\mathrm{I}}$. In the coarse grain region, $\rho_{\mathrm{II}}$ does not increase in proportion to the -1 power of the grain size, but decreases slightly as the grain size increases. It is considered that this is because the free path length of dislocation does not increase infinitely in proportion to the size of the grain size, and therefore does not change significantly in the coarsegrained material.

Figure 13 shows the relationship between $\rho_{\mathrm{II}}$ and $\sigma_{\mathrm{II}} \cdot \sigma_{\mathrm{II}}$ is roughly proportional to the power of $1 / 2$ of $\rho_{\text {II }}$ and follows the Bailey-Hirsch relationship, and in the region where deformation progresses due to plastic deformation, the flow stress of dislocations is determined by the density of other dislocations, i.e., the resistance of dislocation movement is other dislocations.

\section{Conclusions}

In-situ XRD measurements with oscillation of the test piece together with tensile tests enabled measurement of the change in dislocation density during tensile deformation, even in a coarse-grained material with a grain size of $20 \mu \mathrm{m}$. It was thus clarified that the dislocation density changes through four regions, regardless of the grain size of the sample, and it was possible to obtain three parameters, $\sigma_{\mathrm{I}}$, $\sigma_{\mathrm{II}}$, and $\rho_{\mathrm{II}} \cdot \sigma_{\mathrm{I}}$ is the stress at which dislocations begin to multiply, $\rho_{\mathrm{II}}$ is the dislocation density necessary for the deformation to occur only by plastic deformation, and $\sigma_{\mathrm{II}}$ is the stress when the dislocation density reaches $\rho_{\mathrm{II}} . \sigma_{\mathrm{I}}$ and $\sigma_{\mathrm{II}}$ can be regarded as yield stresses with different physical meanings.

Fine-grained materials have a much higher dislocation density $\rho_{\text {II }}$ required for plastic deformation than coarsegrained materials that have been conventionally used for industry. Therefore, region II, where the dislocation density rapidly increases until it reaches $\rho_{\text {II }}$, is wide. In contrast, $\rho_{\text {II }}$ is small for coarse-grained material; therefore, region II is narrow, and the region immediately changes from the elastic deformation region (region I) to the plastic deformation region (region III) and it was not necessary to consider region II. However, it is necessary to understand the mechanical properties of fine-grained materials by considering the existence of region II.

\section{Acknowledgement}

This study was financially supported by the Light Metal Education Foundation Inc., in partial and the support is gratefully appreciated. The Synchrotron radiation experiments were performed at BL19B2 of SPring-8 with the approval of the Japan Synchrotron Radiation Research Institute (JASRI), (Proposal No. 2014B1580, No. 2017A1817 and No. 2018B1588).

\section{REFERENCES}

1) N. Kamikawa, X. Huang, N. Tsuji and N. Hansen: Acta Mater. 57 (2009) 4198-4208.

2) X. Huang, N. Hansen and N. Tsuji: Science 312 (2006) 249-251.

3) H. Adachi, Y. Miyajima, M. Sato and N. Tsuji: Mater. Trans. 56 (2015) $671-678$.

4) H. Adachi, Y. Karamatsu, S. Nakayama, T. Miyazawa, M. Sato and T. Yamasaki: Mater. Trans. 57 (2016) 1447-1453.

5) Y. Miyajima, S. Okubo, T. Miyazawa, H. Adachi and T. Fujii: Philos. Mag. Lett. 96 (2016) 294-304.

6) S. Nakayama, H. Adachi, T. Nabeshima, T. Miyazawa and T. Yamasaki: Sci. Adv. Mater. 8 (2016) 2082-2088.

7) N. Takamasu, T. Miyazawa, Y. Miyajima, H. Adachi and S. Onaka: J. Japan Inst. Met. Mater. 83 (2019) 157-165.

8) G.K. Williamson and R.E. Smallman: Philos. Mag. 8 (1956) 34-45.

9) G.K. Williamson and R.E. Smallman: Acta Crystallogr. 7 (1954) 574 581.

10) T. Ohashi, M. Kawamukai and H. Zbib: Int. J. Plast. 23 (2007) 897914.

11) W.G. Johnston and J.J. Gilman: J. Appl. Phys. 30 (1959) 129-144.

12) H. Adachi, T. Terada and N. Tsuji: J. Jpn. Light Met. 69 (2019) 555561 . 